\title{
Die Vermarktung des Versor-
}

\section{gungsmanagements einer Krankenkasse}

\section{Sind besondere Versorgungsformen für gesunde Versicherte von persönlicher Relevanz?}

Krankenkassen bauen sich zunehmend Versorgungskompetenzen auf. Es stellt sich die Frage, ob besondere Versorgungsangebote auch für gesunde Versicherte von persönlicher Relevanz sind. Krankheit ist ein stochastisches Ereignis. Tritt sie ein, dann liegt in der Regel ein hoher Dringlichkeitsgrad vor. Ein hohes Informationsbedürfnis entsteht bei der Wahl des geeigneten Leistungserbringers und bei der Frage nach Qualität und Angemessenheit der Leistungen. Jedoch ist in dieser Situation die Fähigkeit des Patienten Informationen zu beschaffen und zu verarbeiten stark eingeschränkt. Vor diesem Hintergrund ist es zwar auch für Personen mit einem guten Gesundheitszustand rational, zugleich aber nicht per se zu vermuten, dass sie sich mit der Versorgungskompetenz ihrer Krankenkasse auseinandersetzen, um sich im Krankheitsfall keine Gedanken um eine gute Versorgung machen zu müssen. Aufgabe des Marketing-Managements ist es, eine Verknüpfung der Vorteile besonderer Versorgungsangebote mit den ureigenen Bedürfnissen gesunder Versicherter herzustellen.

\section{Thomas Nebling}

\section{Executive Summary}

Zum 1. Januar 2009 wurde ein Gesundheitsfonds in der gesetzlichen Krankenversicherung (GKV) eingeführt, welcher die Beitragsgelder der Versicherten verwaltet. Hierzu legt die Bundesregierung einen einheitlichen Beitragssatz per Rechtsverordnung fest, womit die kassenindividuellen Beitragssätze entfallen. Die Krankenkassen erhalten zu ihrer Finanzierung aus dem Gesundheitsfonds eine pauschalierte Zuweisung je Versicherten. Kommt eine Krankenkasse mit den Zuweisungen aus dem Gesundheitsfonds nich aus, ist sie gezwungen einen Zusatzbeitrag direkt bei ihren Mitgliedern zu erheben. Übersteigt die Zusatzprämie einen Betrag von 8 EUR, darf die Höhe der Zusatzprämie max. 1 Prozent des beitragspflichtigen Haushaltseinkommens des jeweiligen Mitglieds betragen. Aufgrund dieser 1-ProzentÜberforderungsklausel können gesunde Versicherte mit ei-

Thomas Nebling, Dipl.-Gesundheitsökonom

Techniker Krankenkasse, Hauptverwaltung

Stabsstelle Strategisches Vertrags- und Versorgungsmanagement Hamburg nem hohen Einkommen (sog. gute Risiken) künftig einen noch wichtigeren Faktor für die wirtschaftliche Existenzsicherung einer Krankenkasse spielen. Da in einem solchen Szenario das Preisargument gerade entfällt, gilt es neue Wege zu finden, um für die genannte Zielgruppe attraktiv zu sein. Als Kaufargument soll in dem vorliegenden Beitrag das Versorgungsmanagement einer Krankenkasse diskutiert werden: Wie kann die Zielgruppe der guten Risiken dazu motiviert werden, sich mit den Versorgungsangeboten ihrer Krankenkasse näher zu beschäftigen? Wie gelingt es, dass die genannte Zielgruppe bei ihrer Krankenkasse eine hohe Leistungsstärke bei besonderen Versorgungsformen vermutet? Wie ist es möglich, die Zielgruppe der guten Risiken dazu zu bringen, die Versorgungskompetenz ihrer Krankenkasse als persönlich relevant zu empfinden? Ziel dieser Überlegungen ist es, die Bedeutung des Beitrages bzw. einer Zusatzprämie für gute Risiken als zentrales Auswahlkriterium zu relativieren und dafür die Bedeutung der Versorgungskompetenz einer Krankenkasse als Auswahlkriterium zu erhöhen. Äußern gesunde Versicherte keine Nachfrage nach besonderen Versorgungsangeboten, weil sie bislang den Beitrag für sich als zentrales Kriterium 


\section{THEMA}

ansehen, so ist es die Aufgabe des Marketing-Managements einer Krankenkasse eine Verknüpfung der Vorteile ihrer besonderen Versorgungsangebote mit den (versteckten) Bedürfnissen der gesunden Versicherten vorzunehmen und sie für die Leistungsfähigkeit und Kompetenz ihrer Krankenkasse in Fragen einer optimalen und bedarfsgerechten Versorgung zu interessieren bzw. zu sensibilisieren. Hierzu gilt es eine intelligente Kommunikationsstrategie zu entwickeln. Als Motive für eine persönliche Relevanz des Versorgungsmanagements können das implizite Interesse an der eigenen Gesundheit sowie das implizite Interesse an der Gesundheit nahe stehender Personen (Ehegatten, Kinder, Eltern) angesehen werden. Krankheiten treten in der Regel zufällig und unvorhersehbar auf. Die Wahrscheinlichkeit zu erkranken variiert von Mensch zu Mensch; dass ein Erkrankungsrisiko besteht gilt jedoch für alle (insbesondere akute Erkrankungen und Unfälle), so dass auch gesunde Versicherte potenziell von Krankheit bedroht sind. Wenn eine Erkrankung eintritt, dann weist ihre Behandlung allerdings oft eine hohe Dringlichkeit auf. Mit dem Eintritt einer Erkrankung ist der Patient mit zwei eng miteinander verknüpften Unsicherheiten konfrontiert: Marktunsicherheit und Produktunsicherheit. Marktunsicherheit beschreibt die Unsicherheit hinsichtlich der Wahl eines geeigneten Leistungserbringers. Produktunsicherheit bezieht sich hingegen auf die Unsicherheit hinsichtlich der Angemessenheit und Qualität der erbrachten Leistungen. Aus dem Streben diese Unsicherheiten zu überwinden, resultiert ein Informationsbedürfnis. Jedoch sind die Fähigkeiten zur Beschaffung und Verarbeitung von Informationen gerade dann eingeschränkt (im Krankheitsfall), wenn das Informationsbedürfnis am höchsten ist. Die Notwendigkeit das Informationsbedürfnis zu befriedigen stellt neben der eigentlichen physischen und psychischen Beeinträchtigung einer Krankheit eine weitere Belastung dar. Informationsbedürfnisse zur Bewältigung von Markt- und Produktunsicherheiten entstehen auch im Falle von Vorsorge- und Früherkennungsmaßnahmen, die der Erhaltung der Gesundheit dienen und explizit für gesunde (symptomfreie) Versicherte gedacht sind. So wie der Abschluss einer Krankenversicherung der Absicherung gegen das Erkrankungsrisiko dient, so dient die Frage nach der Versorgungskompetenz einer Krankenkasse der Absicherung gegen die oben beschriebenen Risiken der Markt- und Produktunsicherheit. Die Bausteine des Versorgungsmanagements (Produktportfolio) können analytisch in zwei zentrale Gruppen unterteilt werden: die Organisation, Koordination und Strukturierung von besonderen Versorgungsangeboten (selektives Kontrahieren) sowie die Bereitstellung von Informationsangeboten zur Förderung der Gesundheitskompetenzen für eine effektive und effiziente Nutzung der Angebotsstrukturen (Beratung und Coaching). Die Vermarktung des Versorgungsmanagements gegenüber gesunden Versicherten mit einem hohen Einkommen muss im Rahmen der kommunikationspolitischen Maßnahmen zwei Schritte vollziehen: (1) Den Versicherten von der persönlichen Relevanz des Versorgungsmanagements überzeugen durch die Aktivierung eines Motivs. (2) Dem Versicherten Informtationen über Produkte kommunizieren, welche die (neu) geweckten
Kundenbedürfnisse befriedigen können. Hierzu können jeweils ausgewählte Bausteine des Versorgungsmanagements unterschiedlich miteinander kombiniert werden. Auf diese Weise besteht eine Chance, die Zahlungsbereitschaft gesunder Versicherter mit einem hohen Einkommen zu erhöhen und diese Zielgruppe an die Krankenkasse zu binden.

\section{Problemstellung: Beitrag als zentrales Kriterium gesunder Versicherter?}

In der Versicherungsökonomik werden Versicherungsnehmer zur Vereinfachung klassischerweise in gute Risiken (Personen mit geringer Schadenswahrscheinlichkeit) und schlechte Risiken (Personen mit hoher Schadenswahrscheinlichkeit) unterschieden. In der gesetzlichen Krankenversicherung (GKV) waren die Beiträge bislang allein vom Einkommen des Mitglieds abhängig, so dass hier eine Entkopplung von individuellem Krankheitsrisiko und individueller Beitragshöhe vorliegt. Dies hatte zur Folge, dass tendenziell junge und gesunde Versicherte mit einem hohen Einkommen als gute Risiken galten, da mit diesem Personenkreis die höchsten individuellen Deckungsbeiträge assoziiert wurden. In diesem Kontext erschien es für die Krankenkassen rational, sich um die Akquisition dieser Personenkreise zu bemühen.

Zum 1. Januar 2009 wurde ein Gesundheitsfonds in der GKV eingeführt, der die Beitragsgelder verwaltet. Der Gesundheitsfonds speist sich aus den Beiträgen von Arbeitnehmern und Arbeitgebern sowie aus Steuerzuschüssen des Bundes. Für die Mitgliedsbeiträge legt die Bundesregierung einen einheitlichen Beitragssatz per Rechtsverordnung fest. Damit entfallen die kassenindividuellen Beitragssätze. Die Krankenkassen erhalten zu ihrer Finanzierung aus dem Gesundheitsfonds Versichertenpauschalen, bestehend aus einer Grundpauschale zuzüglich risikoadjustierter (d. h. krankheitsabhängiger) $\mathrm{Zu}$ - und Abschläge. Die Kalkulation dieser Zuweisungen aus dem Gesundheitsfonds basiert auf standardisierten Durchschnittswerten. Reichen die Zuweisungen nicht aus, um die tatsächlichen Ausgaben einer Krankenkasse zu decken, so hat diese die Möglichkeit direkt bei ihren Mitgliedern eine sog. Zusatzprämie zu erheben. Bei der Erhebung einer Zusatzprämie ist eine Überforderungsklausel zu beachten. Übersteigt die Zusatzprämie einen Betrag von 8 EUR, darf die Höhe der Zusatzprämie max. 1 Prozent des beitragspflichtigen Haushaltseinkommens des jeweiligen Mitglieds betragen. Hieraus folgt, dass bei einer Zusatzprämie größer 8 EUR bei jedem Mitglied individuelle Härtefallprüfungen vorzunehmen sind. Im umgekehrten Fall, bei dem die Zuweisungen die tatsächlichen Ausgaben übersteigen, können die Krankenkassen ihre Überschüsse in Form von Prämienausschüttungen an ihre Mitglieder zurückgeben.

Auch wenn durch diese neue Finanzierungs-Systematik versucht wird, eine gewisse risikoäquivalenz der Krankenkassen-Einnahmen herzustellen, können weiterhin gesunde Mitglieder mit einem hohen Einkommen als gute Risiken gelten. Gesunde Mitglieder, die nur geringe 
Leistungsausgaben verursachen, können weiterhin einen positiven Deckungsbeitrag generieren. Bei Versicherten mit einem hohen Einkommen kommt die 1-ProzentÜberforderungsklausel bei der Erhebung einer Zusatzprämie potenziell erst sehr spät zur Entfaltung. Wird aus politökonomischen Gründen eine zu geringe finanzielle Ausstattung des Gesundheitsfonds in Kauf genommen, werden die Krankenkassen gezwungen sein, die fehlenden Mittel über eine Zusatzprämie zu generieren. In einem solchen, als realistisch anzusehenden Szenario, kommt daher den Versicherten mit einem hohen Einkommen eine wichtige Bedeutung beim finanziellen Überlebenskampf der Krankenkassen zu. Dem steht jedoch entgegen, dass die Höhe des Beitrages bislang als das zentrale Kriterium bei der Wahl der Krankenkasse, insbesondere bei jungen und gesunden Versicherten mit einem hohen Einkommen angesehen wurde (Braun/Streich 2003, S. 77 und 83). Hieraus lässt sich die Befürchtung ableiten, dass künftig die Erhebung einer Zusatzprämie diesen Personenkreis zu einer Abwanderung motivieren könnte. Abhängig beschäftigte Versicherte deren beitragspflichtiges Jahresarbeitsentgelt die Versicherungspflichtgrenze (48.600 EUR in 2009) in drei aufeinander folgenden Kalenderjahren überstiegen hat sowie Selbstständige und Beamte haben zudem die Option in die private Krankenversicherung (PKV) abzuwandern. Günstigere Beiträge stehen ebenso bei der Entscheidung für den Wechsel in die PKV an erster Stelle, dicht gefolgt von der Wahrnehmung eines größeren Leistungsumfangs.

Vor diesem Hintergrund stellt sich Frage, wie es einer Krankenkasse gelingen kann, gesunde Versicherte mit einem hohen Einkommen zu gewinnen und zu binden. Da das Preisargument entfällt, gilt es nach Wegen zu suchen, wie die Zahlungsbereitschaft dieser Zielgruppe erhöht werden kann. Als Kaufargument soll in dem vorliegenden Beitrag das Versorgungsmanagement einer Krankenkasse diskutiert werden: Wie kann die Zielgruppe der guten Risiken dazu motiviert werden, sich mit den Versorgungsangeboten ihrer Krankenkasse näher zu beschäftigen? Wie gelingt es, dass die genannte Zielgruppe bei ihrer Krankenkasse eine hohe Leistungsstärke bei besonderen Versorgungsformen vermutet? Wie ist es möglich, die Zielgruppe der guten Risiken dazu zu bringen, die Versorgungskompetenz ihrer Krankenkasse als persönlich relevant zu empfinden? Ziel dieser Überlegungen ist es, die Bedeutung des Beitrages bzw. einer Zusatzprämie für gute Risiken als zentrales Auswahlkriterium zu relativieren und dafür die Bedeutung der Versorgungskompetenz einer Krankenkasse als Auswahlkriterium zu erhöhen.

\section{Aufgaben des Marketing-Managements}

Unter dem Begriff der Vermarktung wird die Verwertung von Güter und Dienstleistungen am Markt verstanden ( $o$. V. 2004). Mit anderen Worten handelt es sich hierbei um den Absatz bzw. den Verkauf von Produkten an Kunden. Grundsätzlich ist das Überleben eines jeden Unternehmens vom gewinnbringenden Absatz seiner Leistungen abhängig. Zwingende Voraussetzung für die Vermarktung eines Angebots ist die Existenz einer entsprechenden Nachfrage. Nach Kotler und Bliemel (1999, S. 10) ist Marketing-Management im wesentlichen als Nachfrage-Management zu verstehen. Fehlt die Nachfrage für ein bestimmtes Angebot, weil die anvisierte Zielgruppe kein Interesse hat oder dem Angebot gleichgültig gegenüber steht, so ist es hier die Aufgabe des Marketing-Managements „Mittel und Wege zu finden, um die Vorzüge des Produkts mit den ureigenen Bedürfnissen und Interessen der Menschen zu verknüpfen“ (Kotler/Bliemel 1999, S. 11). Äußern gesunde Versicherte keine Nachfrage nach besonderen Versorgungsangeboten, weil sie bislang den Beitrag für sich als zentrales Kriterium ansehen, so ist es die Aufgabe des Marketing-Managements einer Krankenkasse eine Verknüpfung der Vorteile ihrer besonderen Versorgungsangebote mit den (versteckten) Bedürfnissen der gesunden Versicherten vorzunehmen.

Die Übermittlung von Informationen wird als Kommunikation bezeichnet. Bruhn (1997, S. 1) definiert Kommunikation als „die Übermittlung von Informationen und Bedeutungsinhalten zum Zwecke der Steuerung von Meinungen, Einstellungen, Erwartungen und Verhaltensweisen bestimmter Adressaten gemäß spezifischer Zielsetzung. “ Möchte eine Krankenkasse neue Versicherte gewinnen bzw. ihre aktuellen Kunden binden, so muss sie diesen Zielgruppen gegenüber Informationen kommunizieren mit dem Zweck, diese von der Krankenkasse so zu überzeugen, dass die Krankenkasse gewählt bzw. nicht gewechselt wird. Alle Maßnahmen, welche Informationen übermitteln mit dem Zweck die Wahrnehmung und das Verhalten einer Zielgruppe zu beeinflussen sind Gegenstand der Kommunikationspolitik (Schmutte 1998, S. 278). Kommunikation kann aus einer informativen sowie auch aus einer beeinflussenden Komponente bestehen und erfüllt daher sowohl Informations- als auch Überzeugungsaufgaben (Meffert 1997, S. 694 f.). Ziel der Kommunikationspolitik einer Krankenkasse ist es, den oben genannten Zielgruppen eine Leistungsanmutung zu signalisieren und ein Leistungsversprechen glaubhaft $\mathrm{zu}$ vermitteln, um sie dazu zu motivieren, eine Bindung mit der Krankenkasse einzugehen bzw. diese fortzuführen.

Um bei der Kundengewinnung erfolgreich zu sein, muss sich eine Krankenkasse genau überlegen, welche Informationen über welches Kommunikationsmedium an welche Zielgruppen kommuniziert werden sollen. Hierzu wird nachfolgend auf die beiden Aspekte Segmentierung und Involvement näher eingegangen. Unter Segmentierung ist nach Freter (1983, S. 17) die Teilung heterogener Gesamtmärkte in homogene Teilmärkte zu verstehen. Dahinter steht das Ziel einen größtmöglichen Markterfolg durch den gezielten Einsatz entsprechender Marketing-Instrumente zu realisieren. Die Segmentierung erfolgt dabei anhand von bestimmten Merkmalen der tatsächlichen bzw. potenziellen Kunden. Nieschlag et al. (1994, S. 583 und $1060 f$.) beschreiben vier Segmentierungskriterien: geographische, biologische, soziodemografische und psychologische Merkmale. Das Informations- und Kommunikationsverhalten der Informationsempfänger wird von diesen 


\section{THEMA}

Merkmalen beeinflusst (Gemünden 1993, S. 1729 ff.). Einen wichtigen Aspekt bei der Kommunikation zielgruppengerechter Informationen stellt das Involvement der Informationsempfänger dar. Involvement definiert Antil (1984, S. 204) als „den Grad wahrgenommener persönlicher Wichtigkeit und/oder persönlichen Interesses, der durch einen Stimulus (oder Stimuli) in einer bestimmten Situation hervorgerufen wird. "Das Involvement ist umso stärker ausgeprägt, je wichtiger die angesprochenen Bedürfnisse und Werte sich für den Informationsempfänger darstellen. Kommuniziert eine Krankenkasse eine Information über ein neues Produkt, so muss diese Information (bzw. das Produkt) für den Versicherten von persönlicher Relevanz sein, damit er sich angesprochen fühlt.

\section{Management der Gesundheitsversorgung}

Die Bedeutung der Gesundheit für das Individuum kann mit Hilfe der Bedürfnispyramide nach Maslow dargestellt werden (Abb. 1).

Dieses Gebilde ordnet menschliche Bedürfnisse nach ihrer Dringlichkeit. Auf der ersten Dringlichkeitsstufe stehen die physischen Bedürfnisse wie Essen, Trinken und Schlafen. Die Befriedigung der physischen Bedürfnisse kann als Voraussetzung zur Befriedigung der Sicherheitsbedürfnisse auf der zweiten Dringlichkeitsstufe angesehen werden. Hierzu zählt insbesondere die Gesundheit. Gesundheit wiederum ist eine wichtige Voraussetzung zur Befriedigung von sozialen, Geltungs- und Selbstverwirklichungsbedürfnissen auf den nachfolgenden Dringlichkeitsstufen. Gesundheit stellt kein Ziel an sich dar, sondern ein Mittel zum Zwecke der Gestaltung eines individuellen und selbstbestimmten Lebens in der Gesellschaft (Bengel/
Strittmatter/Willmann 1998, S. 19). Wer gesund ist, kann am Arbeitsleben teilhaben und seine Arbeitskraft zur Erzielung von Einkommen verwenden, um damit seine Existenz zu sichern. Wer Gesund ist kann selbst für seinen Lebensunterhalt sorgen und bedarf nicht der Unterstützung durch andere in Form von Transferleistungen (Breyer/Zweifel/ Kifmann 2005, S. 74 und 98). Die Erhaltung der Arbeitskraft ist jedoch nur ein Aspekt. So ist Gesundheit vielmehr auch eine notwendige Voraussetzung für die aktive Teilnahme am Gesellschaftsleben und zur Knüpfung von sozialen Kontakten. Sie ermöglicht die Ausübung von Freizeitaktivitäten und ist sowohl die Quelle für eine hohe Lebenserwartung als auch für eine hohe Lebensqualität. Neben Essen, Trinken und Schlafen stellen insbesondere auch medizinische Leistungen einen wichtigen Input zur Erhaltung, Wiederherstellung oder Verbesserung der Gesundheit dar. Breyer, Zweifel und Kifmann (2005, S. 75 ff.) sprechen in diesem Zusammenhang von Gesundheitsproduktion und modellieren Gesundheit als einen (unsichtbaren) Kapitalbestand in den investiert wird und der Leistungen abgibt, also einem Kapitalverzehr unterliegt.

Ausgangspunkt der Produktion von Gesundheitsleistungen ist stets das Streben des Patienten seinen Gesundheitszustand zu erhalten, wiederherzustellen oder zu verbessern. Der Produktionsprozess im Gesundheitswesen kann zwecks Systematisierung in die vier Bereiche Prävention, Kuration, Rehabilitation und Pflege gegliedert werden. Das Ziel der Prävention liegt generell in der Verhinderung einer gesundheitlichen Schädigung. Bei der Kuration geht es um die Behandlung gesundheitlicher Beschwerden. Diese können einen akuten oder chronischen Charakter aufweisen. Einen nach einer Krankheit behinderten Patienten wieder in das Berufsleben und in die Gesellschaft zu integrieren, ist Aufgabe der Rehabilitation. Personen, die wegen Krankheit oder Behinderung für die gewöhnlichen und

\section{Abb. 1: Bedürfnispyramide nach Maslow}

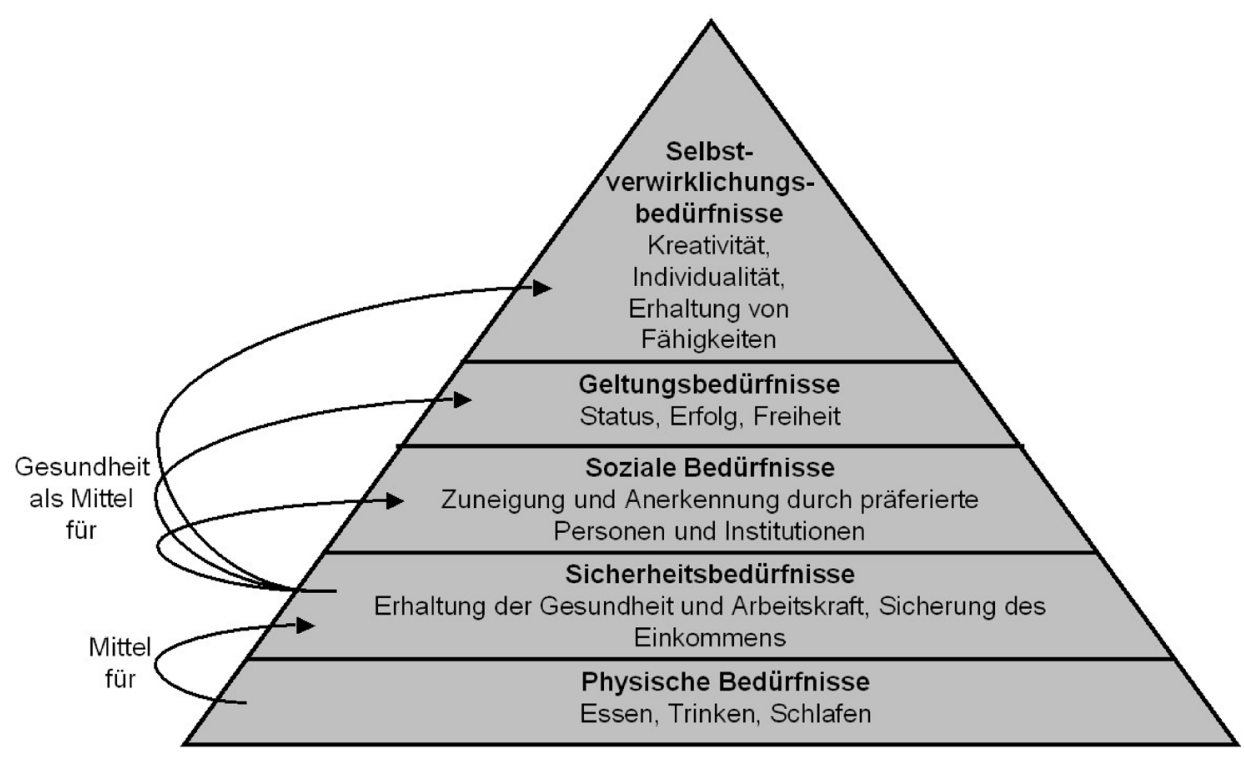


regelmäßig wiederkehrenden Verrichtungen im Ablauf des täglichen Lebens auf Dauer in erheblichem Maße der Hilfe bedürfen gelten als pflegebedürftig. Pflegeleistungen unterstützen die Pflegebedürftigen bei Körperpflege, Ernährung, Mobilität sowie der hauswirtschaftlichen Versorgung.

Das Versorgungsmanagement einer Krankenkasse stellt eine Integration des Vertragsmanagements und des Leistungsmanagements dar. Das Vertragsmanagement regelt die Austauschbeziehungen einer Krankenkasse mit ihren Leistungserbringern. Unter Leistungsmanagement sind alle Maßnahmen zu verstehen, die darauf abzielen, die Leistungsinanspruchnahme der Versicherten zu beeinflussen, um auf eine Erhöhung der Versorgungsqualität hinzuwirken und gleichzeitig Verschwendung zu vermeiden, also die Effizienz der Leistungserbringung zu erhöhen (Spenk 2001). Nach dem Leitbild des mündigen Patienten gewinnen Beratungs- und Coaching-Ansätze innerhalb des Leistungsmanagements zunehmend an Bedeutung. Zweck des Versorgungsmanagements einer Krankenkasse ist die Optimierung der Versorgungsabläufe.

\section{Gesundheitsbezogene Informationsbedürfnisse gesunder Versicherter}

Zweifel und Eisen (2000, S. 7) beschreiben Versicherung als eine Institution, mit deren Hilfe die Unsicherheiten von Wirtschaftssubjekten hinsichtlich zukünftiger Ereignisse reduziert werden können. Unsicherheit, genauer Risiko bzw. Risikoaversion ist die grundlegende Voraussetzung dafür, dass eine Nachfrage nach Versicherung zustande kommt. Mit Blick auf den Gesundheitszustand einer Person besteht hier das Risiko in den mit einer Erkrankung verbundenen Kosten medizinischer Leistungen sowie den durch Arbeitsunfähigkeit bedingten Einkommensausfall. Der Abschluss einer Krankenversicherung hat für den Versicherten unmittelbar den Vorteil, dass er für ein bestimmtes Sicherheitsniveau mit der Versicherungsprämie weniger finanzielle Mittel aufwenden muss im Vergleich zu der erforderlich Rücklage, die gebildet werden müsste, um die Krankheitskosten vollständig selbst zu finanzieren (Freytag 2005, S. 31). Die Frage nach dem Versorgungsmanagement einer Krankenkasse spielt auf die Unterstützung bei der Schadenverhütung (Prävention und Vorsorge), der Früherkennung von Gesundheitsschäden und der Schadenregulierung (Kuration, Rehabilitation) an. Bei gesunden Versicherten wird in der Regel unterstellt, dass der Beitrag das zentrale Kriterium bei der Wahl der Krankenkasse sei. Wie nachfolgend gezeigt wird, ist es auch für gesunde Versicherte rational, sich daneben mit der Versorgungskompetenz ihrer Krankenkasse zu beschäftigen. Hierfür können zwei zentrale Motive identifiziert werden: das Interesse an der eigenen Gesundheit sowie das Interesse an der Gesundheit nahe stehender Personen.

Der Aussage „Ich bin gesund, mich interessiert nur, ob der Beitrag niedrig ist! “ ist mit der Frage „Möchten Sie sich mit der Leistungsfähigkeit Ihrer Krankenkasse erst dann auseinandersetzen wenn Sie krank sind?" zu begegnen. Krankheit ist naturgemäß ein stochastisches Ereignis. Krankheiten treten also in der Regel zufällig und unvorhersehbar auf. Die Wahrscheinlichkeit zu erkranken variiert von Mensch zu Mensch; dass ein Erkrankungsrisiko besteht gilt jedoch für alle (insbesondere akute Erkrankungen und Unfälle). Wenn eine Erkrankung eintritt, dann weist ihre Behandlung allerdings oft eine hohe Dringlichkeit auf (Breyer/Zweifel/Kifmann 2005, S. 178.). Auch wenn der einzelne seinen individuellen Gesundheitszustand beeinflussen kann, so ist es ihm dennoch nicht möglich diesen effektiv zu bestimmen. Neben Umwelteinflüssen wird der individuelle Gesundheitszustand weiterhin vom Zufall bestimmt. Dieser vermag es jederzeit massive Veränderungen herbei zu führen (Breyer/Zweifel/Kifmann 2005, S. 74). Mit dem Eintritt einer Erkrankung ist der Patient mit zwei eng miteinander verknüpften Unsicherheiten konfrontiert: Marktunsicherheit und Produktunsicherheit. Marktunsicherheit beschreibt die Unsicherheit in Bezug auf die Wahl eines geeigneten Leistungserbringers (Gefahr der adversen Selektion). Produktunsicherheit bezieht sich hingegen auf die Unsicherheit in Bezug auf die Angemessenheit und Qualität der erbrachten Leistungen (Bürger 2003, S. 67 f.). Aus dem Streben diese Unsicherheiten zu überwinden resultiert ein Informationsbedürfnis (Unterhuber/Weber 2006, S. 828). Unter der Annahme, dass die Entwicklung des Gesundheitszustandes und die geistigen bzw. kognitiven Fähigkeiten eines Menschen positiv miteinander korreliert sind ergibt sich das Problem, dass die Fähigkeiten zur Beschaffung und Verarbeitung von Informationen gerade dann eingeschränkt sind, wenn das Informationsbedürfnis am höchsten ist (Abb. 2).

Gerade in einer solchen Situation eröffnet sich für die Leistungserbringer ein opportunistischer Handlungsspielraum, der es ihnen ermöglicht Leistungen anzubieten bzw. zu erbringen, welche die Patienten bei besserem Informati-
Abb. 2: Fähigkeit zur Informationsverarbeitung vs. Informationsbedürfnis

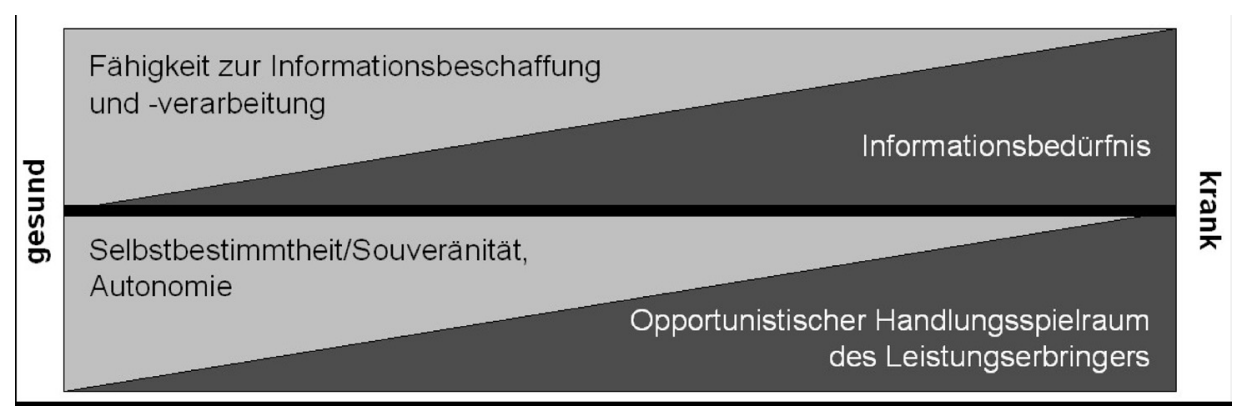




\section{THEMA}

onsstand nicht nachfragen würden. Die Notwendigkeit das Informationsbedürfnis zu befriedigen stellt neben der eigentlichen physischen und psychischen Beeinträchtigung einer Krankheit eine weitere Belastung dar. Vor diesem Hintergrund kann auch dem gesunden Versicherten ein implizites Informationsbedürfnis unterstellt werden. Auch gesunde Versicherte sind dem Risiko zu erkranken ausgesetzt. Daher erwarten auch gesunde Versicherte von ihrer Krankenkasse die bestmögliche Versorgung, für den Fall, dass sie krank werden (Unterhuber/Weber 2006, S. 826). Die Gesundheitsversorgung bezieht sich jedoch nicht allein auf die Behandlung einer Erkrankung (Kuration), sondern weiterhin auch auf die Vermeidung und Früherkennung von Erkrankungen. Bei gesunden Versicherten, die ihre Gesundheit erhalten oder ihren Gesundheitszustand weiter verbessern möchten, kann ein Interesse an Präventionsleistungen und Vorsorgemaßnahmen vermutet werden. Früherkennungsuntersuchungen sind explizit für gesunde Menschen gedacht, die keinerlei Krankheitssymptome aufweisen. Mit diesen Untersuchungen wird das Ziel verfolgt, eine im Entstehen begriffene Erkrankung in einem möglichst frühen Stadium zu erkennen. Damit wird die Hoffnung besserer Heilungschancen bei weniger belastenden Behandlungsprozeduren verbunden. Insbesondere zur Früherkennung von Krebs stehen heute eine Reihe von Untersuchungen (sog. Screenings) zur Verfügung: z. B. Bruskrebs-Screening (Mammografie), HautkrebsScreening, Gebährmutterhalskrebs-Screening, Prostatakrebs-Screening (PSA-Test) und Darmkrebs-Screening (Darmspiegelung). Ferner ist auch etwa an NeugeborenenScreenings und andere zu denken. Vorsorge- und Präventionsmaßnahmen sind nicht immer nützlich und können in bestimmten Fällen sogar einen gesundheitlichen Schaden verursachen. Ebenso sind Früherkennungsmaßnahmen stets mit Nutzen und Risiken verbunden (Mühlhauser 2007). Ein mögliches Risiko einer Früherkennungsuntersuchung besteht etwa in der Gefahr falsch-positiver Befunde, die unnötige aber sowohl psychisch als auch physisch belastende Folgebehandlungen auslösen können (Gigerenzer 2007). Nutzen und Risiken medizinischer Leistungen, nicht nur von Therapien, sondern auch von Präventions-, Vorsorge- und Früherkennungsangeboten, müssen individuell und sorgfältig abgewogen werden. Auch vor diesem Hintergrund kann gesunden Versicherten ein zumindest implizites Informationsbedürfnis über zur Verfügung stehende Versorgungsangebote unterstellt werden.

So wie der Abschluss einer Krankenversicherung der Absicherung gegen das Erkrankungsrisiko dient, so dient die Frage nach der Versorgungskompetenz einer Krankenkasse der Absicherung gegen die Risiken der Markt- und Produktunsicherheit. Dies betrifft nicht nur die eigene Gesundheit, sondern weiterhin auch die Gesundheit nahe stehender Personen. Hierzu gehören insbesondere Familienangehörige, also Ehegatte, Kinder, Eltern und Großeltern. So profitiert ein gesunder Versicherter vom Versorgungsmanagement seiner Krankenkasse, wenn es um die Gesundheitsversorgung seines Ehegatten oder seiner Kinder geht. Die Krankenkasse entlastet dann die Familie von zusätzlichen Belastungen, wie etwa die Suche eines geeigneten Leistungserbringers oder bei der Entscheidung über eine geeignete Behandlungsmethode. Der gleiche Effekt tritt ein, wenn ein gesunder Versicherter seine Eltern oder Großeltern mit einem weniger guten Gesundheitszustand gut versorgt weiß. Dies umso mehr, wenn berufliche Umstände oder räumliche Entfernungen eine persönliche Betreuung durch den Versicherten selbst erschweren. Sind die Familienangehörigen sowie der gesunde Versicherte bei derselben Krankenkasse versichert, so kann ein gutes Versorgungsmanagement einen entsprechenden Kundenbindungseffekt generieren. Zwar ist es aus den genannten Gründen auch für Personen mit einem guten Gesundheitszustand rational, zugleich aber nicht per se zu vermuten, dass sie sich mit der Versorgungskompetenz ihrer Krankenkasse auseinandersetzen, um sich im Krankheitsfall, wie auch bei Vorsorge und Früherkennung, keine Gedanken um eine gute Versorgung machen zu müssen. Die Aufgabe des Marketing-Managements ist es hier, die Zielgruppe der gesunden Versicherten (gute Risiken) für die Leistungsfähigkeit und Kompetenz ihrer Krankenkasse in Fragen einer optimalen und bedarfsgerechten Versorgung zu interessieren bzw. zu sensibilisieren.

\section{Kommunikationspolitische Ansätze des Marketing}

\subsection{Formulierung der Kernkompetenzen einer Krankenkasse}

Aus der in Abschnitt 3 dargestellten Definition des Versorgungsmanagements lassen sich zwei Kernkompetenzen einer Krankenkasse ableiten, aus denen sich wiederum zwei Wertversprechen an die Versicherten formulieren lassen. Die aus dem Vertragsmanagement abzuleitende Kernkompetenz einer Krankenkasse besteht darin, ihren Versicherten nicht nur aus finanzieller sondern vor allem auch aus organisatorischer Sicht Zugang zu einer hochwertigen Gesundheitsversorgung zu verschaffen. Diese Kernkompetenz ergibt sich aus dem Geschäftsmodell des selektiven Kontrahierens, welches die Intermediationsfunktion einer Krankenkasse betont (Nebling 2006). Selektives Kontrahieren bedeutet Vertragsfreiheit. Krankenkassen und Leistungserbringer können jeweils selbst entscheiden mit wem sie einen Versorgungsvertrag abschließen und was in diesen Verträgen im Einzelnen geregelt ist. Die Wertschöpfung einer Krankenkasse kommt hier dadurch zum Ausdruck, dass sie ihren Versicherten besondere Versorgungsangebote zur Verfügung stellt, die sich an deren individuellen Präferenzen und Bedürfnissen orientieren. Es werden Kontakte mit entsprechend geeigneten Leistungserbringern ermöglicht und somit einen Zugang zu besonderen Versorgungsangeboten geschaffen, die sich von der durch Einheitlichkeit geprägten Regelversorgung unterscheiden [1]. Konstituierend hierfür ist ein eigenes Netzwerk aus Leistungserbringern. Notwendige Bedingung, um obiges Wertversprechen zu erfüllen, stellen Aufbau und Pflege eines Netzwerkes an Leistungserbringern (Vertragspartnern) dar. Hinreichende Bedingung sind Aktivitäten zur Förderung der Kontaktaufnahme zwischen Versicher- 
ten und Leistungserbringern. Konkret schafft eine Krankenkasse dadurch einen Wert für ihre Versicherten, indem sie für diese die Vorauswahl (Selektion) und Qualitätsprüfung von Leistungserbringern übernimmt sowie Preise, Behandlungsstandards, Gewährleistungsansprüche und Garantien für die Leistungserstellung verhandelt.

Die aus dem Leistungsmanagement - genauer aus dem Handlungsfeld Beratung und Coaching - abzuleitende Kernkompetenz besteht darin, den Versicherten beim Erwerben und Aufrechterhalten von Gesundheitskompetenzen zu unterstützen. Gesundheitskompetenzen beschreiben die Fähigkeiten von Individuen im täglichen Leben informierte und selbstbestimmte Entscheidungen zu treffen, welche die Gesundheit positiv beeinflussen. Diese Entscheidungen werden zu Hause, am Arbeitsplatz, im Gesundheitssystem, auf Märkten sowie in der Gesellschaft getroffen. Daher sind Gesundheitskompetenzen jeweils relevant für die Rolle der Individuen als Patienten, Versicherte, Konsumenten und Bürger. Hieraus lassen sich fünf Gesundheitskompetenz-Bereiche ableiten (Kickbusch 2006, S. 69 ff.):

- Kompetenzbereich persönliche Gesundheit: Hierzu zählen Grundkenntnisse und Wissen über Gesundheit, über die Anwendung von gesundheitsförderlichem, gesundheitsbewahrendem und krankheitsverhindertem Verhalten sowie über Selbstpflege.

- Kompetenzbereich Systemorientierung: Dies beinhaltet die Fähigkeit erfolgreich durch die Komplexität des Gesundheitssystems navigieren zu können sowie selbstbestimmt und kompetent gegenüber Leistungserbringern und anderen Akteuren im Gesundheitswesen aufzutreten.

- Kompetenzbereich Konsumverhalten: Hier geht es um jene Fähigkeiten, bei Konsum- und Dientsleistungsentscheidungen auch gesundheitliche Aspekte zu berücksichtigen sowie auch im Zweifel seine Verbraucherrechte einklagen und durchsetzen zu können. von Gesundheitsinformationen sowie zu einer kritischen Prüfung auf deren persönlichen Relevanz und Evidenz (Wahrheitsgehalt). Die Strukturen im deutschen Gesundheitswesen wie auch die Beschaffenheit von Gesundheitsdienstleistungen gelten als hoch komplex. Komplexität erzeugt Intransparenz, Verunsicherung, Hilflosigkeit und damit auch Unzufriedenheit. Die Förderung von Gesundheitskompetenzen durch die Bereitstellung von Beratungsund Coachingangeboten können als einen Schlüssel zur Überwindung bzw. Beherrschung dieser Komplexitäten angesehen werden. Gesundheitskompetente Menschen sind in der Lage, eine Krankheit zu verstehen und sie im täglichen Leben bewältigen zu können. Sie sind ferner dazu in der Lage, ihre Interessen stärker gegenüber den Leistungserbringern zu vertreten, besser mit diesen $\mathrm{zu}$ interagieren sowie sich aktiv an medizinischen Entscheidungsprozessen zu beteiligen (Kranich 2005). Durch den Aufbau einer Krenkompetenz im Handlungsfeld Beratung und Coaching, schafft eine Krankenkasse dadurch einen Wert für ihre Versicherte, dass sie diese darin unterstützt, selbstbestimmte und informierte Entscheidungen bei Fragen der eigenen Gesundheit sowie der eigenen Lebensqualität treffen zu können. Diese Kompetenzen können wiederum auch mit Blick auf die Gesundheit nahe stehender Personen relevant sein. Die oben beschriebenen Kompetenzbereiche zeigen zudem, dass Gesundheitskompetenzen nicht nur in der Situation einer aktuen oder chronsichen Erkrankung wertvoll sein können.

Die Bausteine des Versorgungsmanagements (Produktportfolio) können auf der Basis der beiden skizzierten Kernkompetenzen einer Krankenkasse analytisch entsprechend in zwei zentrale Gruppen unterteilt werden: die Organisation, Koordination und Strukturierung von besonderen Versorgungsangeboten (selektives Kontrahieren) sowie die Bereitstellung von Informationsangeboten zur Förderung der Gesundheitskompetenzen für eine effektive und effiziente Nutzung der Angebotsstrukturen (Beratung und Coaching) (Abb. 3).

- Kompetenzbereich Gesundheitspolitik: Dies beinhaltet die Fähigkeit, sich als informierter Bürger zu aktuellen gesundheitspolitischen Fragen eine eigene Meinung zu bilden und entsprechend zu handeln, z. B. bei politischen Wahlen oder durch aktive Mitgliedschaften in Patientenorganisationen.

- Kompetenzbereich Arbeitswelt: Hierzu zählen die Fähigkeiten Arbeitsunfälle und Berufskrankheiten zu vermeiden sowie sich für Sicherheit am Arbeitsplatz und gesundheitsförderliche Arbeitsbedingungen zu engagieren.

Gesundheitskompetenzen können durch Maßnahmen der Gesundheitsbildung gefördert werden. Diese dienen der Aneignung von Kompetenzen zur Beschaffung, Bearbeitung und Nutzung

Abb. 3: Bausteine des Versorgungsmanagements

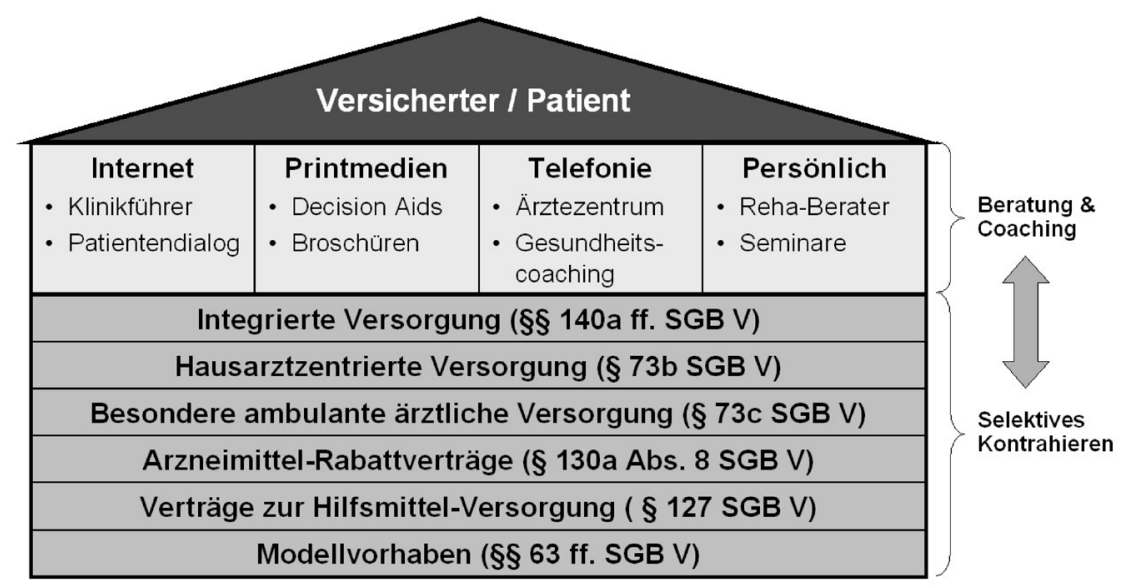




\section{THEMA}

Der Gesetzgeber hat den Krankenkassen inzwischen eine Reihe von Instrumenten zur individuellen Gestaltung des Vertragsmanagements zur Verfügung gestellt:

- Die integrierte Versorgung stellt die bislang meist genutze Vertragsoption dar. Mit diesem Vertragsansatz wird das Ziel verfolgt, die in der Regelversorgung separierten Leistungssektoren (ambulant hausärztlich, ambulant fachärztlich, stationär, Rehabilitation) so miteinander zu verknüpfen, dass Sektoren übergreifende Versorgungsprozesse entstehen, die sich stärker an den Problemen und Bedürfnissen der Patienten orientieren.

- Die hausarztzentrierte Versorgung (sog. Hausarztmodell) zielt auf die Etablierung eines Gate-Keeper-Systems ab. Hier fungiert der Hausarzt als Lotse des Patienten. Patienten, die an einem Hausarztmodell teilnehmen möchten, müssen sich bereit erklären, immer zuerst ihren Hausarzt aufzusuchen, der sie dann bei Bedarf zu einem Facharzt oder in eine Klinik überweist.

- Die besondere ambulante ärztliche Versorgung emöglicht es den Krankenkasse individuelle Versorgungsverträge mit ausgewählten Ärzten oder Ärztevereinigungen abzuschließen, wahlweise über das gesamte Spektrum der ambulanten ärztlichen Versorgung (Haus- und Facharztversorgung) oder ausgewählte Bereiche hiervon.

- Im Rahmen von Arzneimittel-Rabattverträgen und Hilfsmittel-Verträgen können Krankenkassen eigene Verträge zur Sicherstellung der Versorgung ihrer Versicherten mit Arznei- und Hilfsmitteln abschließen.

- Im Rahmen von Modellvorhaben können Krankenkassen Leistungen zur Verhütung und Früherkennung von Krankheiten sowie zur Krankenbehandlung, die nach den Regelungen des Sozialgesetzbuches (noch) keine Leistungen der GKV sind durchführen oder mit ausgewählten Leistungserbringern vereinbaren. Die Erprobung neuer Untersuchungs- und Behandlungsmethoden (Versorgungsforschung) muss wissenschaftlich evaluiert werden.

Das Handlungsfeld Beratung und Coaching verfolgt insgesamt drei Ziele: (1) Information der Versicherten über vorhandene besondere Versorgungsangebote, (2) Vermittlung von Kompetenzen, diese Angebotsstrukturen effektiv und effizient zu nutzen, sich darin orientieren zu können, mit den Leistungserbringern partnerschaftlich interagieren und die eigenen Präferenzen in den medizinischen Entscheidungsprozess mit einbringen zu können sowie (3) Vermittlung von Kompetenzen, sich auch außerhalb dieser Strukturen (zu Hause, am Arbeitsplatz etc.) mit seiner Krankheit arrangieren bzw. auf eine gute Gesundheit hinwirken zu können (Selbstmanagement). Hierbei können verschiedene Medien zum Einsatz kommen (Straub/Nebling/Müller 2008; Straub/Nebling 2009; Dierks 2009):

- Internet: (1) Suchmaschinen im Internet für die Suche nach geeigneten Leistungserbringern (Suchkriterien z. B. Wohnort, Art der Erkrankung etc.) und Hinterlegung von dataillierten Informationen über die jeweiligen Anbieter. (2) Interaktiver, web-basierter Patientendialog zur Vorbereitung eines Arztgespräches, Frage-AntwortRhythmus: System merkt sich die Eingaben des Nutzers und blendet nur relavante Informationen hierzu auf. Vermittlung von Wissen über Entstehung, Behandlung und Umgang mit einer bestimmten Erkrankung sowie Ermittlung der eigenen Präferenzen hinsichtlich der zur Verfügung stehenden Therapiealternativen.

- Printmedien: (1) Decision Aids (Entscheidungshilfen) informieren den Patienten über potenzielle Nutzen und Risiken einer bestimmten medizinischen Maßnahme. Unterstützung bei der selbstbestimmten Entscheidungsfindung für oder gegen die Inanspruchnahme einer bestimmten Leistung (z. B. Brustkrebs-Screening). (2) Broschüren und Flyer mit Informationen über besondere Versorgungsangebote und Versorgungsverträge.

- Telefonie: (1) Servicenummer, bei der speziell geschulte Ärzte rund um die Uhr medizinische Auskünfte geben (Ärztezentrum). Bereitstellung einer verlässlichen, ergänzenden und gut erreichbaren Informationsquelle. (2) Telefonisches Gesundheitscoaching mit Informationen und Hintergründen zur Erkrankung des Versicherten. Frühzeitige Identifizierung von Symptomen und ggf. Empfehlung, den behandelnden Arzt aufzusuchen, um Eskalationen im Krankheitsverlauf zu vermeiden. Unterstützung bei der Umsetzung persönlicher Ziele im Alltag (Selbstmanagement) zur Erhöhung der Lebensqualität und Bewahrung der Selbstständigkeit.

- Persönlich: (1) Reha-Berater (Case-Manager) als persönliche Ansprechpartner vor Ort mit sozialversicherungsrechtlichem und medizinischem Know-how koordinieren den Behandlungsverlauf, Unterstützen das Treffen selbstbestimmter Entscheidungen und geben eine Orientierung im Gesundheitswesen. (2) Seminare und Kurse zur Förderung der Gesundheitsbildung. Vermittlung allgemeiner Kenntnisse über das Gesundheitssystem, Wissen über allgemeine medizinische Zusammenhänge sowie krankheitsspezifisches Wissen.

Beratungs- und Coachingangebote können einerseits als Maßnahmen verstanden werden, die den selektiven Vertragsangeboten vorgelagert sind, indem sie die Versicherten auf die Nutzung der selektiv-vertraglichen Angebotsstrukturen vorbereiten. Andererseits können Beratungs- und Coachingangebote als Maßnahmen verstanden werden, die den selektiven Vertragsangeboten nachgelagert sind, indem sie die Versorgung durch selektiv kontrahierte Leistungserbringer ergänzen.

\subsection{Formulierung einer Vermarktungsstrategie}

Die Vermarktung des Versorgungsmanagements gegenüber gesunden Versicherten mit einem hohen Einkommen muss im Rahmen der kommunikationspolitischen Maßnahmen zwei Schritte vollziehen: (1) Den Versicherten von der persönlichen Relevanz des Versorgungsmanagements überzeugen durch die Aktivierung eines Motivs. (2) Dem Versicherten Informtationen über Produkte kommunizieren, welche die (neu) geweckten Kundenbedürfnisse 
befriedigen können. Wie bereits in Abschnitt 3 erläutert sind vier Motive denkbar:

(1) Bedürfnis nach einer guten Versorgung im Falle einer unerwarteten Erkrankung aus Interesse an der eigenen Gesundheit.

(2) Bedürfnis nach einer guten Versorgung zur Vermeidung einer Erkrankung aus Interesse an der eigenen Gesundheit.

(3) Bedürfnis nach einer guten Versorgung im Falle einer unerwarteten Erkrankung aus Interesse an der Gesundheit einer nahestehenden Person.

(4) Bedürfnis nach einer guten Versorgung zur Vermeidung einer Erkrankung aus Interesse an der Gesundheit einer nahestehenden Person.

Die Produkte zur Befriedigung dieser (neu) geweckten Kundenbedürfnisse ergeben sich aus den Bausteinen des Versorgungsmanagements. Beide Dimensionen können zu einer Entscheidungsmatrix zusammen geführt werden (Abb. 4). Mit Hilfe dieser Matrix lässt sich festlegen, welches Bedürfnis mit welchem Produkt in der Kundenansprache kombiniert werden soll. Voraussetzung ist eine vorangegangene Segmentierung der Zielgruppe nach dem prioritär anzusprechenden Bedürfnis (Motiv) und den jeweils für die Bewerbung am besten geeigneten Produktkategorien.

In Abhängigkeit davon, wie stark die jeweiligen Motive aktiviert werden können, sollte die Versichertenansprache entweder konkrete Produkte bewerben oder auf einer abstrakteren Ebene ganze Produktgruppen. Je schwächer ein Motiv aktiviert werden kann, desto eher bietet es sich an, die generelle Existenz individueller Versorgungsangebote auf Basis selektiver Verträge oder Beratungs- und Coachingangebote zu bewerben, um damit einen allgemeinen Mehrwert (value added service) zu betonen. Je stärker ein Motiv aktiviert werden kann, desto eher bietet es sich an, einzelne konkrete Produkte, z. B. Versorgungsverträge am Wohnort des Versicherten, zu betonen. Vor dem Hintergrund der letzten Gesundheitsreformen in Deutschland wächst die Skepsis gegenüber dem künftigen Versorgungsniveau in der GKV. Im Rahmen der Kommunikationsstrategie gilt es daher, häufig geäußerte Ängste und subjektive Wahrnehmungen der Bevölkerung hinsichtlich „Versorgungslücken“ und „Zwei-Klassen-Medizin“ aufzufangen und subjektive Sicherheit durch die Versorgungskompetenz der Krankenkasse zu erzeugen. Das implizite gesundheitsbezogene Sicherheitsbedürfnis auch gesunder Versicherter mit einem hohen Einkommen kann demnach die Vermarktung des Versorgungsmanagements einer Krankenkasse unterfüttern.

Die Wahrnehmung, dass die Leistungen aufgrund gesetzlicher Regelungen bei allen Krankenkassen gleich seien, ist in der Bevölkerung weit verbreitet (Braun/Streich 2003, S. 73, 77 und 83). Auch wenn die Leistungskataloge überall nahezu identisch sein mögen, so bietet die Art und Weise der Leistungserbringung bzw. Leistungsgewährung doch erhebliches Differenzierungspotenzial. Möglichkeiten einer Differenzierung entspringen dabei den Bausteinen des Versorgungsmanagements. Im Wettbewerb gilt es für die Versicherten fühl- und erlebbare Unterschiede zu anderen Krankenkassen herauszustellen. Dem Argument „Die Leistungen sind bei allen Krankenkassen gleich!“ ist mit dem Gegenargument „Die Tatsache, dass viele Leistungen von allen Krankenkassen bezahlt werden, sagt noch nichts darüber aus, wie die Leistungserbringung im Einzelfall organisiert und koordiniert wird! “ zu begegnen. Mit Blick auf die Zielgruppe gesunder Versicherter mit einem hohen Einkommen ist es weiterhin erforderlich in der Kommunikation auch Vorteile gegenüber der PKV zu betonen. Nachfolgend werden Beispiele für Werbebotschaften beschrieben, welche den Mehrwert einer Krankenkasse mit Versorgungskompetenz betonen und auf eine Erhöhung der Zahlungsbereitschaft abzielen.

\section{„Machen Sie sich jetzt Gedanken über Ihre Kranken- versicherung, damit Sie sich im Krankheitsfall keine} Gedanken über Ihre Versorgung machen müssen! " Diese Aussage spielt auf die in Abhängigkeit des Gesundheitszustandes unterschiedlich stark ausgeprägten Fähigkeiten Informationen beschaffen und verarbeiten zu können an (,Wir Entlasten Sie von Ihrer Sorge um eine gute Versorgung im Krankheitsfall! "). Hier liegt auch ein Wettbewerbsvorteil gegenüber der PKV: Konflikte zwischen Privatversicher-
Abb. 4: Kommunikationspolitische Entscheidungsmatrix

\begin{tabular}{|c|c|c|c|c|c|c|}
\hline & \multicolumn{4}{|c|}{ Motive für persönliche Relevanz } \\
\hline & & & \multicolumn{2}{|c|}{$\begin{array}{l}\text { Interesse an der } \\
\text { eigenen Gesundheit }\end{array}$} & \multicolumn{2}{|c|}{$\begin{array}{l}\text { Interesse an der Gesundheit einer } \\
\text { nahestehenden Person }\end{array}$} \\
\hline & & & $\begin{array}{l}\text { Behandlung } \\
\text { Erkrankung }\end{array}$ & $\begin{array}{l}\text { Vorsorge und } \\
\text { Früherkennung }\end{array}$ & $\begin{array}{l}\text { Behandlung } \\
\text { Erkrankung }\end{array}$ & $\begin{array}{l}\text { Vorsorge und } \\
\text { Früherkennung }\end{array}$ \\
\hline \multirow{5}{*}{ 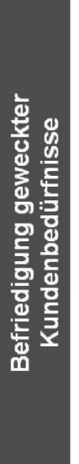 } & \multirow{4}{*}{ 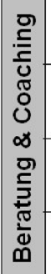 } & Internet & & & & \\
\hline & & Printmedien & & & & \\
\hline & & Telefonie & & & & \\
\hline & & Persönlich & & & & \\
\hline & \multicolumn{2}{|c|}{ 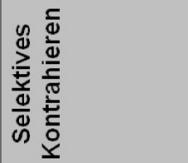 } & & & & \\
\hline
\end{tabular}


ten und ihrem Versicherer entstehen am häufigsten bei der Frage nach der medizinischen Notwendigkeit einer Behandlung. Dies betrifft insbesondere die Frage, ob eine stationäre Krankenhausbehandlung erforderlich war. Wird dies aus Sicht der Versicherer verneint, lehnen diese eine entsprechende Kostenübernahme im Anschluss an die Behandlung ab (Ombudsmann 2007, S. 8 f.). Bei einer gesetzlichen Krankenkasse besteht die Unsicherheit bezüglich der Kostenübernahme in dieser Form nicht. Bei eigenen kassenindividuellen Versorgungsangeboten werden in der Regel klare medizinische Kriterien definiert, die erfüllt sein müssen, damit ein Patient ein bestimmtes Angebot in Anspruch nehmen kann. Über diese Kriterien werden die Patienten bereits im Voraus von ihrer Krankenkasse bzw. ihrem behandelden Arzt aufgeklärt. Der selektive Versorgungsvertrag, der keine Vorfinanzierung einer Behandlung durch den Patienten vorsieht, stellt daher eine Art „Finanzierungsgarantie" dar.

\section{„Reicht es Ihnen, wenn Ihre Krankenversicherung nur die Arztrechnungen bezahlt? Wir bieten mehr! "Diese Aussa-} ge deutet einen Mehrwert gegenüber der PKV an, welche keine Versorgungsverträge mit den Leistungserbringern vorhält. Das Geschäftsmodell des selektiven Kontrahierens bietet den gesetzlich Versicherten einen Wert, den die PKV nicht generieren kann. Während sich die PKV lediglich auf die Absicherung gegen die finanziellen Folgen einer Krankheit beschränkt, bieten gesetzliche Krankenkassen mit Versorgungskompetenz darüber hinausgehende Leistungen zur Überwindung von Markt- und Produktunsicherheiten an. In diesem Zusammenhang kann ein weiterer Vorteil gegenüber der PKV beschrieben werden: Der PKV wird häufig der vermeintliche Vorteil eines im Vergleich zur GKV größeren Leistungsumfanges nachgesagt. Dies ist jedoch zu relativieren. So ist bekannt, dass an Privatpatienten oft mehr und umfangreichere Untersuchungen vorgenommen werden. Diese Mengenausweitung ist jedoch nicht zwangsläufig mit einem besseren Behandlungsergebnis oder Gesundheitszustand verbunden. Im Gegenteil finden sich immer wieder Beispiele, bei welchen eine Mengenausweitung der Leistungen über den Bereich des medizinisch Sinnvollen und Sicheren hinaus stattfindet - zum Schaden der Patienten (Heier 2007; Drexler 2007). Auch in der Medizin gilt die Erkenntnis „Weniger ist oft mehr" bzw. „Das Teuerste ist nicht immer das Beste“. Dieser Gefahr wirken selektive Versorgungsverträge von gesetzlichen Krankenkassen mit Hilfe von entsprechenden finanziellen Anreizstrukturen sowie vereinbarten Behandlungsstandards und Leitlinein entgegen. Gegenstand von selektiven Versorgungsverträgen kann auch die Erprobung neuer Untersuchungs- und Behandlungsmethoden sein. Ziel ist es hier, den Versicherten so bald als möglich einen Zugang zu neuen medizinischen Verfahren zu verschaffen, die sowohl sinnvoll als auch sicher und effizient sind (Müller 2007). Hinter der Forcierung von kontrollierten Einführungen neuer medizinischer Verfahren als Markenattribut steckt weiterhin die Botschaft, dass die Krankenkasse ihre Versicherten vor experimentellen (unsicheren bzw. gefährlichen) Verfahren schützt. Dies stellt ein weiteres Differenzierungsmerkmal gegenüber der PKV dar, wel- che den Eindruck erweckt für die uneingeschränkte (und unkontrollierte?) Einführung von Innovationen zu werben (Schmitt 2006; PKV 2007).

\section{Fazit}

Kommt eine Krankenkasse mit den Zuweisungen aus dem Gesundheitsfonds nicht aus, ist sie gezwungen einen Zusatzbeitrag direkt bei ihren Mitgliedern zu erheben. Aufgrund der 1-Prozent-Überforderungsklausel können gesunde Versicherte mit einem hohen Einkommen künftig einen noch wichtigeren Faktor für die wirtschaftliche Existenzsicherung einer Krankenkasse spielen. Da in einem solchen Szenario gerade ein niedriger Preis als Wettbewerbsparameter entfällt, gilt es neue Wege zu finden, um für die genannte Zielgruppe attraktiv zu sein. In diesem Beitrag wurde diskutiert, inwiefern das Versorgungsmanagement einer Krankenkasse die Zahlungsbereitschaft gesunder Versicherte mit einem hohen Einkommen erhöhen könnte. Als Motive für eine persönliche Relevanz wurde das implizite Interesse an der eigenen Gesundheit sowie das implizite Interesse an der Gesundheit nahe stehender Personen dargestellt. Krankheit ist ein stochastisches Ereignis, so dass auch gesunde Versicherte potenziell davon bedroht sind. Beim Eintritt einer Erkrankung entstehen Informationsbedürfnisse zur Bewältigung von Markt- und Produktunsicherheiten. Dies gilt ebenfalls für Vorsorge- und Früherkennungsmaßnahmen, die der Erhaltung der Gesundheit dienen und explizit für gesunde (symptomfreie) Versicherte gedacht sind. Vor diesem Hintergrund ist es auch für gesunde Versicherte rational, jedoch nicht per se zu vermuten, dass sie sich mit der Versorgungskompetenz ihrer Krankenkasse beschäftigen. Aufgabe des MarketingManagements ist es, die oben genannten Motive bei gesunden Versicherten zu aktivieren und aufzuzeigen, wie die Angebote des Versorgungsmanagements diese Bedürfnisse befriedigen können. Auf diese Weise besteht eine Chance, die Zahlungsbereitschaft gesunder Versicherter mit einem hohen Einkommen zu erhöhen und diese Zielgruppe an die Krankenkasse zu binden.

\section{Literatur}

Antil, J. H. (1984), Conceptualization and Operationalization of Involvement, in: Kinnear, T. (Hrsg.), Advances in Consumer Research XI, Provo (Utah), S. 203 - 209

Bengel, J./Strittmatter, R./Willmann, H. (1998), Was erhält Menschen gesund? Antonovskys Modell der Salutogenese - Diskussionsstand und Stellenwert, Bundeszentrale für gesundheitliche Aufklärung, Köln.

Börgel, A. (2003), Zielgruppenorientierte Übermittlung krankheitsbezogene Informationen an Versicherte der Gesetzlichen Krankenversicherung, elektronische Veröffentlichung, http://elib.ub.uni-osnabrueck.de/ publications/diss/E-Diss313_thesis.pdf, Abruf am 23.02.2007.

Braun, B./Streich, W. (2003), Gutes Beitrags-Leistungs-Verhältnis oder Sicherheit - was wollen die Bürger von ihrer Krankenkasse?, in: Böcken, J./ Braun, B./Schnee, M. (Hrsg.), Gesundheitsmonitor 2003 - Die ambulante Versorgung aus Sicht von Bevölkerung und Ärzteschaft, Gütersloh.

Breyer, F./Zweifel, P./Kifmann, M. (2005), Gesundheitsökonomik, 5. Auflage, Berlin.

Bruhn, M. (1997), Kommunikationspolitik - Grundlagen der Unternehmenskommunikation, München.

Bürger, C. (2003), Patientenorientierte Information und Kommunikation im Gesundheitswesen, Wiesbaden. 
Dierks, M.-L. (2009), Angebot und Nachfrage nach kritischer Gesundheitsbildung - Erfahrungen aus der ersten Patientenuniversität in Deutschland, in: Klusen, N./Fließgarten, A./Nebling, T. (Hrsg.), Informiert und selbstbestimmt - Der mündige Bürger als mündiger Patient, BadenBaden, S.379-393.

Drexler, S. (2007), Ärztlicher Rohrreinigungsdienst - Ethik und Monetik in der Kardiologie, in: Frankfurter Allgemeine Sonntagszeitung vom 22.08.2007.

Freter, H. (1983), Marktsegmentierung, Stuttgart.

Freytag, A. (2005), Risikodifferenzierung in der Krankenversicherung, Baden-Baden.

Gemünden, H. G. (1993), Information: Bedarf, Analyse und Verhalten, in: Grochla, E./Wittmann, W. (Hrsg.), Handwörterbuch der Betriebswirtschaft, Stuttgart, S. 1725-1735.

Gigerenzer, G. (2007), Das Einmaleins der Skepsis - Über den richtigen Umgang mit Zahlen und Risiken, 3. Auflage, Berlin

Heier, M. (2007), In den Klauen der Halbgötter, in: Frankfurter Allgemeine Sonntagszeitung vom 24.06.2007.

Kickbusch, I. (2006), Die Gesundheitsgesellschaft - Megatrends der Gesundheit und deren Konsequenzen für Politik und Gesellschaft, Gamburg.

Kolter, P./Bliemel, F. (1999), Marketing-Management, 9. Auflage, Stuttgart.

Kranich, C. (2005), Patientenkompetenz, in: Helmert, U./Schumann, H./ Jansen-Bitter, H. (Hrsg.), Souveräne Patienten? Die Wiederentdeckung des Patienten im 21. Jahrhundert, Augsburg, S. 35-49.

Meffert, H. (1997), Marketing Management - Analysen, Strategien, Implementierung, Wiesbaden.

Mühlhauser, I. (2007), Ist Vorbeugen besser als Heilen?, in: Zeitschrift für ärztliche Fortbildung und Qualitätsmanagement im Gesundheitswesen, Jg. 101, S. 293-299.

Müller, H. (2007), Mythos Innovation? Anmerkungen zu medizintechnischen Entwicklungen aus Sicht der Gesetzlichen Krankenversicherung, in: Groß, D./Jakobs, E.-M.(Hrsg.), E-Health und technisierte Medizin Neue Herausforderungen im Gesundheitswesen, Berlin, S. 101-111.

Nebling, T. (2006), Das Geschäftsmodell des selektiven Kontrahierens - Eine Analyse wertschöpfender Aktivitäten gesetzlicher Krankenkassen, in: Zeitschrift für die gesamte Versicherungswissenschaft, Nr. 4/2006, S. 655-683.

Nieschlag, R./Dichtel, E./Hörschgen, H. (1994), Marketing, 17. Auflage, Berlin.

Ombudsmann der Privaten Kranken- und Pflegeversicherung (2007), Tätigkeitsbericht 2006, http://www.pkv-ombudsmann.de/dokumente/PKVOmb-TB-2006.pdf, Abruf am 04.09.2007.

o. V. (2004), Stichwort „Vermarktung“, in: Gabler Wirtschaftslexikon, 16. Auflage, Wiesbaden, S. 3143.

PKV (2007), Privatversicherte profitieren erheblich häufiger von neuen Arzneimitteln, Verband der privaten Krankenversicherung e.V., Pressemitteilung vom 5. März 2007.

Schmitt, J. M. (2006), Chancen für Innovationen nutzen!, in: PKV Publik, Nr. 7/2006 vom 15.09.2006.

Schmutte, A. (1998), Total Quality Management im Krankenhaus, Wiesbaden.

Spenk, C. (2001), Leistungssteuerung in der gesetzlichen Krankenversicherung: Ein Paradigmenwechsel, in: Sozialer Fortschritt, Jg. 50, Nr. 8, S. $199-200$

Straub, C./Nebling, T./Müller, H. (2008), Translating research into practice: A German sickness fund supporting patient participation, in: Patient Education an Counseling, Vol. 73, S. 544-550.

Straub, C./Nebling, T. (2009), Vom Patienten zum Partner - Die Versorgungsstrategie der Techniker Krankenkasse, in: Klusen, N./Fließgarten, A./ Nebling, T. (Hrsg.), Informiert und selbstbestimmt - Der mündige Bürger als mündiger Patient, Baden-Baden, S. 394-412.

Unterhuber, H./Weber, S. (2006), Die neue Rolle der Krankenkassen im Wettbewerb, in: Rebscher, H. (Hrsg), Gesundheitsökonomie und Gesundheitspolitik im Spannungsfeld zwischen Wissenschaft und Politikberatung, Heidelberg, S. 819-838.

Zweifel, P./Eisen, R. (2000), Versicherungsökonomie, Berlin.

\section{Fußnoten:}

[1] Die Regelversorgung in der gesetzlichen Krankenversicherung wird über sog. Kollektivverträge zwischen den Verbänden der Krankenkassen und den Verbänden der Leistungserbringer organisiert. Da diese Kollektivverträge dem Ausschließlichkeitsprinzip folgen sind individuelle Differenzierungen einzelner Anbieter grundsätzlich nicht möglich.

\section{Der „Klassiker“}

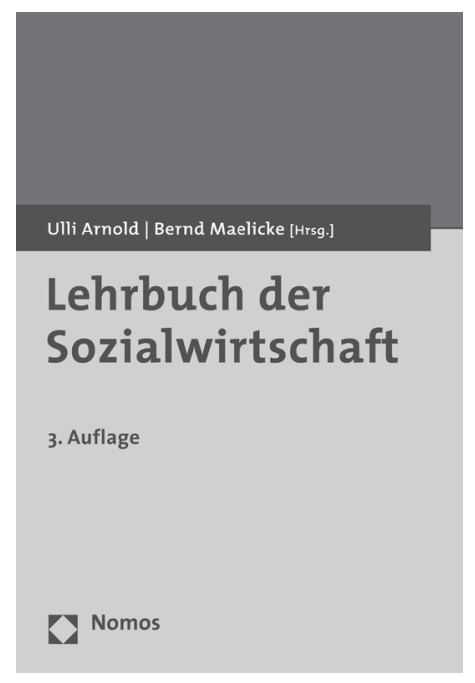

\section{Lehrbuch der Sozialwirtschaft}

Herausgegeben von Prof. Dr. Dr. h.c. Ulli Arnold und Prof. Dr. Bernd Maelicke

$$
\begin{aligned}
& \text { 3. Auflage 2009, } 826 \text { S., brosch., 49,- } € \text {, } \\
& \text { ISBN 978-3-8329-2680-9 }
\end{aligned}
$$

Die rapide Veränderung der politischen und strukturellen Rahmenbedingungen für die Sozialwirtschaft erfordert grundlegende Änderungen in der Ausbildung und Weiterbildung von Führungskräften sozialer Dienstleistungsorganisationen. Die vollständig aktualisierte und neu bearbeitete 3. Auflage vermittelt Basiswissen und aktuelle Informationen zu den wichtigsten Themen.

\section{"Das aktualisierte Lehrbuch ist als Grundwerk zur Vermittlung von Basiswissen zu zentralen thematischen Schwerpunkten der Sozialwirt- schaft und des Sozialmanagements hervor- ragend geeignet."}

Prof. Dr. Gabriele Moos, wwww.socialnet.de, zur Vorauflage

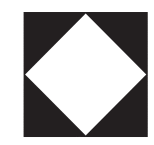

Nomos

Bitte bestellen Sie im Buchhandel ode versandkostenfrei unter $\downarrow$ www.nomos-shop.de 